\title{
Examining the Emergency Medical Treatment and Active Labor Act: impact on telemedicine for neurotrauma
}

\author{
Sanjit Shah, MD,, George L. Yang, MD,, Diana T. Le, MS, ${ }^{2}$ Christina Gerges, BS, ${ }^{3}$ \\ James M. Wright, MD, ${ }^{3,4}$ Ann M. Parr, MD, PhD, ${ }^{5}$ Joseph S. Cheng, MS, MD, ${ }^{1,2}$ and \\ Laura B. Ngwenya, MD, PhD',2
}

'Department of Neurological Surgery, University of Cincinnati College of Medicine; 2University of Cincinnati College of Medicine, Cincinnati; ${ }^{3}$ Case Western Reserve University School of Medicine, Cleveland; ${ }^{4}$ Department of Neurosurgery, Cleveland Clinic, Cleveland, Ohio; and ${ }^{5}$ Department of Neurosurgery, University of Minnesota, Minneapolis, Minnesota

\begin{abstract}
The Emergency Medical Treatment and Active Labor Act (EMTALA) protects patient access to emergency medical treatment regardless of insurance or socioeconomic status. A significant result of the COVID-19 pandemic has been the rapid acceleration in the adoption of telemedicine services across many facets of healthcare. However, very little literature exists regarding the use of telemedicine in the context of EMTALA. This work aimed to evaluate the potential to expand the usage of telemedicine services for neurotrauma to reduce transfer rates, minimize movement of patients across borders, and alleviate the burden on tertiary care hospitals involved in the care of patients with COVID-19 during a global pandemic. In this paper, the authors outline EMTALA provisions, provide examples of EMTALA violations involving neurosurgical care, and propose guidelines for the creation of telemedicine protocols between referring and consulting institutions.
\end{abstract}

https://thejns.org/doi/abs/10.3171/2020.8.FOCUS20587

KEYWORDS EMTALA; coronavirus; COVID-19; traumatic brain injury; TBI; subarachnoid hemorrhage; telemedicine; neurotrauma

$\mathrm{T}$ HE Emergency Medical Treatment and Active Labor Act (EMTALA) was passed by the US Congress in 1986 with the intent to protect patient access to emergency medical treatment regardless of insurance or socioeconomic status. The original intent of this legislation was to prevent a phenomenon known as "dumping," in which patients are transferred from private to public hospitals due to insurance status. Although originally brief by contemporary standards at a mere 4 pages, this law is now one of the most comprehensive and significant pieces of legislation preventing hospitals from denying patient access to emergency medical treatment on the basis of socioeconomic factors. ${ }^{1}$ As EMTALA has expanded, a number of telemedicine-oriented provisions have been added to ensure that patient protections are preserved. Although the legislation has been enforced by the Centers for Medicare and Medicaid Services (CMS), with numerous institutions being fined or investigated for violations, very little literature or precedent exists regarding the use of telemedicine for stabilization of patients presenting to the Emergency Department (ED) with regard to compliance with EMTALA.

With the outbreak of the disease caused by the SARSCoV-2 virus, more commonly known as COVID-19, healthcare in the US saw a dramatic acceleration in the adoption and use of telemedicine. Although nonurgent medical concerns have been addressed in an outpatient setting with telemedicine, patients suffering traumatic injuries, such as traumatic brain injury (TBI), have continued to present to the hospital for evaluation.

Traumatic subarachnoid hemorrhage (tSAH) occurs in up to $82 \%$ of TBIs every year, and nearly 1.7 million

ABBREVIATIONS CMS = Centers for Medicare and Medicaid Services; ED = Emergency Department; EMC = emergency medical condition; EMTALA = Emergency Medical Treatment and Active Labor Act; MSE = medical screening examination; $\mathrm{mTBI}=$ mild TBI; OIG = Office of the Inspector General; SDH = subdural hematoma; TBI = traumatic brain injury; $\mathrm{tSAH}=$ traumatic subarachnoid hemorrhage.

SUBMITTED June 30, 2020. ACCEPTED August 18, 2020.

INCLUDE WHEN CITING DOI: 10.3171/2020.8.FOCUS20587. 
people suffer from mild TBI (mTBI) annually in the US. ${ }^{2}$ Although tSAH is a common entity in patients with polytrauma, the majority of patients with isolated tSAH do not require admission to the hospital and are often discharged home without any intervention. ${ }^{2-8}$ Despite the literature reporting a low rate of complications from tSAH, patients are often transferred for further evaluation and management to a Level I trauma center by facilities that lack inhouse neurosurgical consultation or experience with TBI due to fear of neurological deterioration. Although exact cost figures vary based on location, these transfers represent a tremendous cost to the healthcare system, ${ }^{6,9}$ with some studies demonstrating significant cost savings with adoption of nontransfer protocols. Patients with TBI represent a unique population in which telemedicine may be used for evaluation and management while complying with EMTALA guidelines due to geographic availability of neurosurgeons.

The purpose of this study was to evaluate the potential to expand the usage of telemedicine in neurotrauma, specifically mTBI, within the confines of EMTALA. Here, we outline the salient aspects of EMTALA and provide examples of EMTALA violations involving neurosurgical care. We also propose a telemedicine protocol for the management of mTBI.

\section{EMTALA Provisions}

Passed as an unfunded mandate, EMTALA was initially enacted in response to widespread concern regarding a process in which some hospitals committed financial or socioeconomic discrimination against certain patients by "dumping" the uninsured on public hospitals. ${ }^{10,11}$ The legislation sought to address this problem by requiring adequate medical screening and medical stabilization of patients with emergency medical conditions (EMCs) before discharge. EMTALA requires hospitals with Medicare/ Medicaid funding to do the following. ${ }^{10-12}$

- Provide an adequate medical screening examination (MSE) to include or exclude an EMC.

- Stabilize the patient's EMC(s) medically to the best of the hospital's capabilities to the point where their condition will not decline upon transfer.

- Provide timely consultation, treatment, and hospitalization for the EMC(s) within the capacity of the treating hospital and medical staff by maintaining a list of oncall physicians.

- Transfer patients to a facility providing a higher level of care if necessitated by the EMC(s) and if the benefits outweigh the risks. If the receiving hospital has specialized capabilities greater than the referring hospital, they must accept all patients for transfer unless there is no physical space in the hospital or necessary equipment is not functioning.

- Report known violations by hospitals and physicians for noncompliance with the legislation.

It is the hospital and referring provider's responsibility under EMTALA to guarantee timely consultation for treatment versus transfer of patients. The referring provider has the ability to determine whether the on-call physician needs to physically see the patient in the hospital. If deemed necessary, the on-call physician must evaluate the patient; otherwise it is an EMTALA violation on the part of both the consultant and the hospital.

EMTALA provides civil penalties if violated and is enforced by the Office of the Inspector General (OIG) for the Department of Health and Human Services (DHHS), of which the CMS is a part. The law defines an EMC as "a medical condition manifesting itself by acute symptoms of sufficient severity (including severe pain) such that the absence of immediate medical attention could reasonably be expected to result in placing the health of the individual in serious jeopardy or serious dysfunction of any bodily organ or part." 12 Failure to adhere to the above stipulations may result in CMS civil penalties for both the hospital and the provider. Additionally, although patients are only allowed to file lawsuits against hospitals for EMTALA violations, physicians may be individually sued for malpractice. Many of the provisions listed under EMTALA are now nationally considered the standard of care due to its widespread enforcement, and as such, physicians found to be in violation of EMTALA may reasonably be found guilty of malpractice.

Although full data regarding the number of alleged EMTALA violations, hospital terminations, and enforcement actions are lacking, the OIG and CMS have published incomplete data on this topic. Between 2002 and 2015, CMS performed 527 investigations of potential EMTALA violations. ${ }^{11}$ Of these investigations, an estimated 167 to 192 resulted in settlements, with an average reported fine of US\$31,733..$^{11,13}$ Notably, the number of EMTALA-related settlements steadily trended downward during that period of time. This was an improvement from the period of time between 1994 and 1998, when the total number of settled violations per year remained relatively stable, with an average of 164 confirmed settlements per year. ${ }^{13,14}$ Between 2013 and 2020, the OIG reported 81 EMTALA settlements, 4 of which involved cases that required neurosurgical consultation (Fig. 1). ${ }^{14}$ Two allegations were made due to failure of the treating hospital to provide an adequate MSE, provide stabilizing treatment, and make the decision to transfer to a higher level of care in a timely fashion. The other two were due to refusal to accept appropriate transfers (Table 1). ${ }^{14}$

Increased use of telemedicine between institutions could provide an avenue to allow treating hospitals to provide timely consultation and for receiving hospitals to deny inappropriate transfers, without risk of EMTALA violation. Adoption of telemedicine has also been demonstrated to reduce unnecessary transfers and decrease the time to necessary neurosurgical intervention. ${ }^{6,15,16}$ Reducing unnecessary transfers may also prevent a sense of "fatigue" in receiving hospitals and lower the risk of refusal for appropriate transfers. In the subspecialty of neurotrauma, patients with mTBI are frequently subject to overtriage to higher levels of care. Protocols to allow hospitals to manage patients with mTBI in the absence of a consulting neurosurgeon have been derived. ${ }^{17,18}$ The ability to overcome geographic and personnel limitations with telemedicine can increase access to neurosurgical experts who can assist in evaluation, treatment, and decision-making to enable nonspecialist providers to manage mTBI with con- 


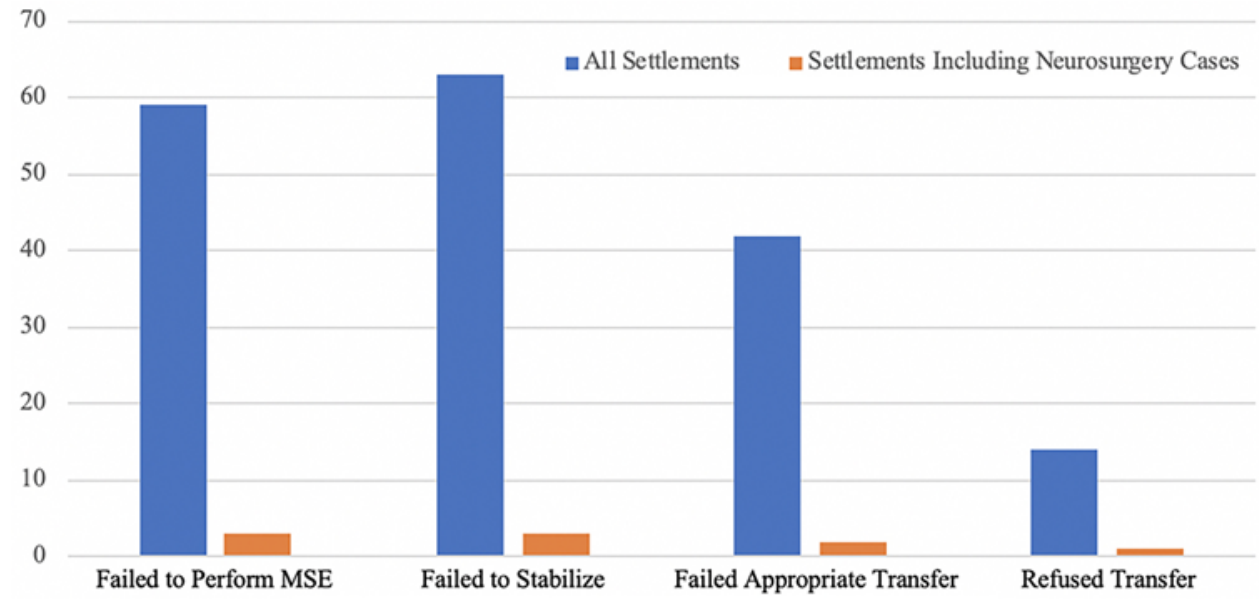

FIG. 1. OIG-reported EMTALA settlements from 2013 to 2020. Settlements involving violations that revolved around neurosurgical cases are indicated in orange, and total settlements are noted in blue. The numbers on the $y$-axis represent the number of cases.

fidence. With this in mind, we propose a set of guidelines to lay the groundwork for adoption of telemedicine-based nontransfer protocols between institutions.

\section{Proposed Guidelines for Telemedicine}

The acceleration of telemedicine in the COVID-19 era provides an opportunity for significant systemic change in neurotrauma on a macrocosmic scale. ${ }^{19-21}$ Prior to COVID-19, multiple studies had already evaluated the use of telemedicine in mTBI and demonstrated both safety and financial efficacy. Dario et al. used telemedicine to provide neurotrauma care to surrounding community medical centers by having neurosurgeons at tertiary care centers review transmitted clinical data..$^{22}$ The authors found that $72 \%$ of neurosurgeons responded to consults within the first hour and the implementation of this protocol resulted in an $84 \%$ decrease in the number of patients requiring transfers. ${ }^{22}$ Medeiros de Bustos et al. evaluated a neurological emergency telemedicine network over 14 years and found that for 23,710 patients, interhospital transfer rates were reduced by $50 \%$ without a significant increase in mortality among nontransferred patients. In addition, they saved US $\$ 4.2$ million over the 14-year study period in associated costs. ${ }^{9}$ Levy et al. report that the approximate cost of transfer in their region was US\$3018, with savings of approximately US $\$ 38,228$ per year over their 6-year study period. ${ }^{6}$ Although no studies exist to demonstrate a benefit in reducing the spread of COVID-19 by increasing the use of telemedicine, it follows logically that reducing the rates of transfer will also reduce the amount of movement of family members for visitation and transport.

The literature supporting conservative management in

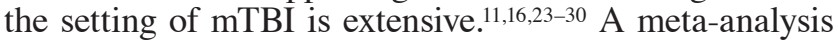
demonstrated that the need for neurosurgical intervention in patients with mTBI and isolated tSAH was $0.0017 \%$,

TABLE 1. OIG-reported EMTALA settlements including patients requiring neurosurgical evaluation or intervention

\begin{tabular}{|c|c|c|c|c|}
\hline Reported Violation & $\begin{array}{l}\text { Failed to } \\
\text { Provide } \\
\text { MSE }\end{array}$ & $\begin{array}{l}\text { Failed to Provide } \\
\text { Stabilizing } \\
\text { Treatment }\end{array}$ & $\begin{array}{l}\text { Failed to Provide } \\
\text { Appropriate } \\
\text { Transfer }\end{array}$ & $\begin{array}{l}\text { Refused to Accept } \\
\text { Appropriate } \\
\text { Transfer }\end{array}$ \\
\hline $\begin{array}{l}\text { 1. Hospital in North Carolina did not diagnose a patient with a surgery-related } \\
\text { SDH and the patient was discharged. Additionally, hospital failed to transfer a } \\
\text { patient with SAH secondary to aneurysmal rupture to an appropriate facility. }\end{array}$ & Yes & Yes & Yes & No \\
\hline $\begin{array}{l}\text { 2. Kentucky hospital discharged a patient without CT head scan. Patient } \\
\text { returned to ED and was found to have multiple surgery-related skull fractures; } \\
\text { was transferred promptly at that time. }\end{array}$ & Yes & Yes & Yes & No \\
\hline $\begin{array}{l}\text { 3. Hospital in Ohio failed to accept a patient with acute-on-chronic SDH when } \\
\text { they had capacity and an in-house neurosurgeon was available. }\end{array}$ & No & No & No & Yes \\
\hline $\begin{array}{l}\text { 4. Alabama hospital neurosurgeon was called about an SDH requiring emer- } \\
\text { gency hemicraniectomy. Refused transfer because he believed that the } \\
\text { patient was brain dead, despite being told that the patient was just intubated } \\
\text { and paralyzed. Patient was transferred to another facility where they received } \\
\text { surgical intervention and were discharged to a rehabilitation facility in } 5 \text { days. }\end{array}$ & No & No & No & Yes \\
\hline
\end{tabular}


with only 1 death attributed to neurological injury per 15,327 evaluated patients. Studies by Ashkenazi et al. and Levy et al. have demonstrated both the safety and costeffectiveness of managing select patients with mTBI and associated traumatic intracerebral hemorrhage with telemedicine consultations. ${ }^{6,16}$

Using the groundwork established by these prior studies, we believe that institutions may be able to use the following items as framework for instituting telemedicine consultations to decrease unnecessary transfers of patients with mTBI.

1. Factors to consider for patient inclusion:

a. Mild TBI (Glasgow Coma Scale score 13-15)

b. Head CT demonstrating any of the following findings:

i. Minimal or small tSAH

ii. Punctate or minimal contusion/intraparenchymal hemorrhage $\left(<10 \mathrm{~cm}^{3}\right)^{2,7,8}$

iii. Thin subdural hematomas (SDHs) without mass effect

2. Factors to consider for patient exclusion:

a. Moderate to severe TBI (Glasgow Coma Scale score 3-12)

b. Presence of coagulopathy (defined by use of an antiplatelet or anticoagulant, liver insufficiency, or known history of hemophilia)

c. Evidence of a significant mechanism of injury (e.g., basilar skull fractures, rhinorrhea, otorrhea, suspected CSF leaks)

d. Spontaneous intracerebral hemorrhage

e. Head CT demonstrating either of the following:

i. Presence of an SDH $>5 \mathrm{~mm}$ in size

ii. Presence of an epidural hematoma

3. The transferring hospital must be able to share radiological imaging for review by the consultant neurosurgeon in a timely fashion.

4. Telemedicine video consultation technology should be available to allow the consultant to examine the patient, if requested.

5. At the conclusion of the telemedicine consultation, decisions regarding need and timing of repeat imaging and criteria for escalation of care and transfer need to be established.

6. The agreement has to be honored with reciprocal hospital-approved policies, to minimize the risk of an EMTALA violation if it is perceived that an appropriate transfer was refused or a timely transfer was delayed.

\section{Barriers to Telemedicine in Neurotrauma}

There are a number of barriers to the widespread adoption of telemedicine services, such as concerns with potential medicolegal vulnerabilities, including liability and credentialing. Herein we limit the discussion to liability with regard to federal regulations set by EMTALA. As demonstrated above, EMTALA violations within the field of neurosurgery included cases in which there was either a failure to appropriately stabilize and transfer or failure to accept a patient in whom neurosurgical intervention was warranted. These violations of federal law can put neu- rosurgeons and their institutions at risk for nonmonetary sanctions, fines, and civil lawsuits. At this time, there is not a uniform standard of care for the implementation of telemedicine in neurotrauma.

As the practice of telemedicine grows, so do medicolegal risks associated with virtual care. However, this is an area in which EMTALA itself is particularly ambiguous, because telemedicine consultation was not yet widely available when the Act was instituted as law. As of 2004, additional guidelines were issued that allowed for the use of telemedicine services when patients were unable to be physically assessed due to geography.$^{31}$ Other permissible situations include when patients initially present to hospitals located in areas in which there is a professional shortage or counties outside of metropolitan statistical areas. ${ }^{31}$ This concept was reinforced by a 2020 CMS memo regarding EMTALA after the COVID-19 outbreak that stated, "Hospitals may use telehealth equipment to perform the MSE by qualified medical personnel. The qualified medical personnel may be on campus (and using telehealth to self-contain) or offsite (due to staffing shortages)." ${ }^{32}$

The primary means of ensuring EMTALA compliance while implementing telemedicine programs is incorporating clear clinical details into the service contracts between institutions in order to define the division of labor and liability. ${ }^{33}$ EMTALA requires policies governing the use of telemedicine providers to be set in writing, with specific details regarding the scope of services that can be provided and transfer protocols. It is in the service contract that agreements regarding liability can be decided upon in advance. Ideally, both parties would share mutual responsibility for instituting appropriate patient care. By encouraging shared, equitable responsibility, we hope to increase participation from consulting neurosurgeons and to establish improved and timely access to a knowledgeable expert, with the option to transfer if necessary. Implementation of protocols using our proposed framework can improve the initial evaluation and stabilization of patients and streamline the interfacility transfer process, while reassuring providers and institutions that they remain compliant with EMTALA.

Other barriers to adoption of telemedicine include technical and logistical difficulties between different healthcare systems. Regional protocols, hospital agreements, and technology both to facilitate the video call and to easily share imaging between the referring and consulting provider are all necessary before telemedicine protocols can be implemented.

\section{Conclusions}

EMTALA protects access to emergency care for all patients regardless of socioeconomic status, while leaving room for the adoption of new technologies. The COVID-19 pandemic has resulted in an accelerated adoption of telemedicine services across medical specialties and settings. The national response to this pandemic has created many exceptions that have allowed for this rapid adoption of new practices. Neurotrauma specialists should take this opportunity to establish pathways and guidelines to expand the availability of neurosurgical services where there is 
inadequate coverage due to the geographical distribution of neurosurgeons. If implemented properly, telemedicine protocols would allow for appropriate evaluation, triage, reassessment, and disposition of such patients while reducing the cost of transfer and increasing the comfort of the referring hospital emergency physicians. If done properly, such protocols can be implemented while ensuring compliance with EMTALA.

\section{References}

1. Zibulewsky J. The Emergency Medical Treatment and Active Labor Act (EMTALA): what it is and what it means for physicians. Proc (Bayl Univ Med Cent). 2001;14(4):339-346.

2. Nassiri F, Badhiwala JH, Witiw CD, et al. The clinical significance of isolated traumatic subarachnoid hemorrhage in mild traumatic brain injury: a meta-analysis. J Trauma Acute Care Surg. 2017;83(4):725-731.

3. Marincowitz C, Lecky FE, Townend W, et al. The risk of deterioration in GCS13-15 patients with traumatic brain injury identified by computed tomography imaging: a systematic review and meta-analysis. J Neurotrauma. 2018;35(5): 703-718.

4. Lewis PR, Dunne CE, Wallace JD, et al. Routine neurosurgical consultation is not necessary in mild blunt traumatic brain injury. J Trauma Acute Care Surg. 2017;82(4):776-780.

5. Lee JJ, Segar DJ, Asaad WF. Comprehensive assessment of isolated traumatic subarachnoid hemorrhage. J Neurotrauma. 2014;31(7):595-609.

6. Levy AS, Orlando A, Salottolo K, et al. Outcomes of a nontransfer protocol for mild traumatic brain injury with abnormal head computed tomography in a rural hospital setting. World Neurosurg. 2014;82(1-2):e319-e323.

7. Yun BJ, White BA, Benjamin Harvey H, et al. Opportunity to reduce transfer of patients with mild traumatic brain injury and intracranial hemorrhage to a Level 1 trauma center. Am J Emerg Med. 2017;35(9):1281-1284.

8. Yun BJ, Borczuk P, Wang L, et al. Evaluation of a low-risk mild traumatic brain injury and intracranial hemorrhage emergency department observation protocol. Acad Emerg Med. 2018;25(7):769-775.

9. Medeiros de Bustos E, Berthier E, Chavot D, et al. Evaluation of a French regional telemedicine network dedicated to neurological emergencies: a 14-year study. Telemed $J$ E Health. 2018;24(2):155-160.

10. Rosenbaum $\mathrm{S}$. The enduring role of the emergency medical treatment and active labor act. Health Aff (Millwood). 2013; 32(12):2075-2081.

11. Zuabi N, Weiss LD, Langdorf MI. Emergency Medical Treatment and Labor Act (EMTALA) 2002-15: Review of Office of Inspector General patient dumping settlements. West $J$ Emerg Med. 2016;17(3):245-251.

12. Centers for Medicare \& Medicaid Services. Medicare Program; Clarifying policies related to the responsibilities of Medicare-participating hospitals in treating individuals with emergency medical conditions. Accessed September 4, 2020 https://www.cms.gov/Regulations-and-Guidance/Legislation/ EMTALA/Downloads/CMS-1063-F.pdf

13. McKenna RM, Purtle J, Nelson KL, et al. Examining EMTALA in the era of the Patient Protection and Affordable Care Act. AIMS Public Health. 2018;5(4):366-377.

14. HHS Office of Inspector General. Civil monetary penalties and affirmative exclusions. Accessed September 4, 2020. https://oig.hhs.gov/fraud/enforcement/cmp/cmp-ae.asp

15. Jackson EM, Costabile PM, Tekes A, et al. Use of telemedicine during interhospital transport of children with operative intracranial hemorrhage. Pediatr Crit Care Med. 2018;19(11): $1033-1038$.
16. Ashkenazi I, Haspel J, Alfici R, et al. Effect of teleradiology upon pattern of transfer of head injured patients from a rural general hospital to a neurosurgical referral centre. Emerg Med J. 2007;24(8):550-552.

17. Joseph B, Friese RS, Sadoun M, et al. The BIG (brain injury guidelines) project: defining the management of traumatic brain injury by acute care surgeons. J Trauma Acute Care Surg. 2014;76(4):965-969.

18. Martin GE, Carroll CP, Plummer ZJ, et al. Safety and efficacy of brain injury guidelines at a Level III trauma center. $J$ Trauma Acute Care Surg. 2018;84(3):483-489.

19. Hollander JE, Carr BG. Virtually perfect? Telemedicine for Covid-19. N Engl J Med. 2020;382(18):1679-1681.

20. Klein BC, Busis NA. COVID-19 is catalyzing the adoption of teleneurology. Neurology. 2020;94(21):903-904.

21. Ohannessian R, Duong TA, Odone A. Global telemedicine implementation and integration within health systems to fight the COVID-19 pandemic: a call to action. JMIR Public Health Surveill. 2020;6(2):e18810.

22. Dario C, Scannapieco G, Scienza R, et al. The neurosurgical telecounseling network in the Veneto Region: 4 years of experience of HEALTH OPTIMUM. Telemed J E Health. 2014;20(11):1009-1014.

23. Sweeney TE, Salles A, Harris OA, et al. Prediction of neurosurgical intervention after mild traumatic brain injury using the national trauma data bank. World J Emerg Surg. 2015; 10(1):23.

24. Borczuk P, Penn J, Peak D, Chang Y. Patients with traumatic subarachnoid hemorrhage are at low risk for deterioration or neurosurgical intervention. J Trauma Acute Care Surg. 2013; 74(6):1504-1509.

25. Phelan HA, Richter AA, Scott WW, et al. Does isolated traumatic subarachnoid hemorrhage merit a lower intensity level of observation than other traumatic brain injury? $\mathrm{J} \mathrm{Neu-}$ rotrauma. 2014;31(20):1733-1736.

26. Borczuk P. Predictors of intracranial injury in patients with mild head trauma. Ann Emerg Med. 1995;25(6):731-736.

27. Carlson AP, Ramirez P, Kennedy G, et al. Low rate of delayed deterioration requiring surgical treatment in patients transferred to a tertiary care center for mild traumatic brain injury. Neurosurg Focus. 2010;29(5):E3.

28. Clement CM, Stiell IG, Schull MJ, et al. Clinical features of head injury patients presenting with a Glasgow Coma Scale score of 15 and who require neurosurgical intervention. Ann Emerg Med. 2006;48(3):245-251.

29. Fabbri A, Servadei F, Marchesini G, et al. Antiplatelet therapy and the outcome of subjects with intracranial injury: the Italian SIMEU study. Crit Care. 2013;17(2):R53.

30. Haydel MJ, Preston CA, Mills TJ, et al. Indications for computed tomography in patients with minor head injury. N Engl J Med. 2000;343(2):100-105.

31. Brown LC, Cochran RG. CMS issues much-anticipated EMTALA guidelines. J Trauma Nurs. 2004;11(3):107-110.

32. Centers for Medicare \& Medicaid Services. Emergency Medical Treatment and Labor Act (EMTALA) Requirements and Implications Related to Coronavirus Disease 2019 (COVID-19). Accessed September 4, 2020. https://www.cms. gov/medicareprovider-enrollment-and-certificationsurveycertificationgeninfopolicy-and/emergency-medical-treatmentand-labor-act-emtala-requirements-and-implications-relatedcoronavirus

33. Rockwell KL, Gilroy A. Emergency telemedicine: achieving and maintaining compliance with the Emergency Medical Treatment and Labor Act. Telemed J E Health. 2018;24(11): 934-937.

\section{Disclosures}

The authors report no conflict of interest concerning the materi- 
Shah et al.

als or methods used in this study or the findings specified in this paper.

\section{Author Contributions}

Conception and design: Yang, Shah, Ngwenya. Acquisition of data: Yang, Shah. Analysis and interpretation of data: Shah. Drafting the article: Yang, Shah, Le. Critically revising the article: all authors. Reviewed submitted version of manuscript: all authors. Approved the final version of the manuscript on behalf of all authors: Yang. Administrative/technical/material support: Yang, Ngwenya. Study supervision: Parr, Cheng, Ngwenya.

\section{Correspondence}

George L. Yang: University of Cincinnati College of Medicine, Cincinnati,OH.yangge@ucmail.uc.edu. 